Proceedings

\title{
A Hypothesis of Autism Approached with the Nonlinear Model ${ }^{+}$
}

\author{
Xu Huang and Jun Meng * \\ Center for Data Mining and Systems Biology, College of Electrical Engineering, Zhejiang University, \\ Hangzhou 310027, China; 21610138@zju.edu.cn \\ * Correspondence: junmeng@zju.edu.cn \\ + Presented at the IS4SI 2017 Summit DIGITALISATION FOR A SUSTAINABLE SOCIETY, Gothenburg, \\ Sweden, 12-16 June 2017.
}

Published: 8 June 2017

\begin{abstract}
This paper combined the current brain science development; put forward a novel hypothesis for the pathogenesis of autism which is called Double-Mirror Reflection Model. The camera-monitor experiment's results and theoretical deduction show that the hypothesis is consistent with the current brain biology cognition and discovery of autism. This hypothesis will provide a new idea for the prevention and diagnosis of autism by the methods of information ecology.
\end{abstract}

Keywords: autism; positive feedback; information ecology

\section{Introduction}

With Autism Spectrum Disorder(ASD) , also known as autism, the patients will appear the phenomena of language disorder, communicating disorder or social disorder who are mostly two or three-year-old boy [1]. The pathogenesis of autism is not yet clear. People began to study the etiology of autism from 1943, and have ruled out education and family factors; the consensus is that the cause of autism is mainly caused by brain biology factors. Therefore, this paper intends to propose a hypothesis of autism from the perspective of a nonlinear system, as well as a model to explain this hypothesis, hoping to unify the current phenomenon and Essence of autism, and finally providing a new thinking for the current autism study.

\section{Hypothesis and Experiments}

Brain neurons are also similar to "exhaustion", the evolution of species phenomenon [2,3]. It also can be called Neuron Plasticity [4]. For causality, one explanation is that because of the abnormal structure of the brain leading to abnormal synapses and the formation of pathological neurological loops, so that patients have obstacles of communication and understanding or repetitive behaviors.

In order to explain this phenomenon, this paper presents a "double-mirror reflection" model, as Figure 1 shows: autism is a kind of erroneous iteration of the brain of autistic patients, a positive feedback process of behavior and structure, the patient is not in chaos (which is controllable), but into the cyclical activities. 


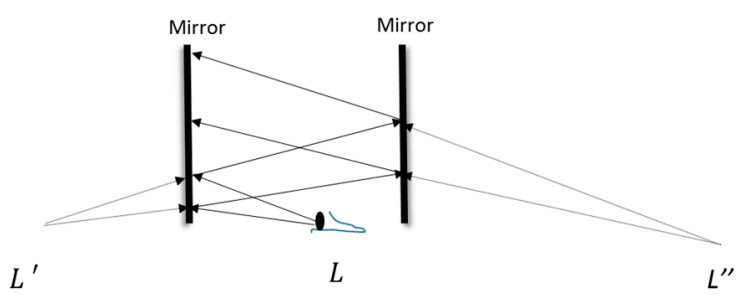

Figure 1. Assuming that we have an observer placed in the center of the mirror, using the observer to observe the phenomenon in the mirror; multi-layer mapping will be find in the mirrors.

To explain this hypothesis, we designed the screen-camera experiment as a simple closed loop in Figure 2.

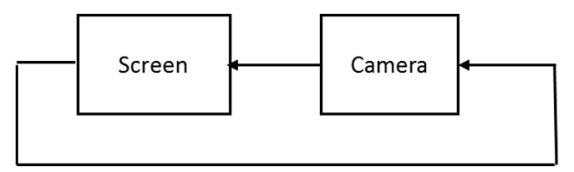

Figure 2.The closed-loop system is composed by camera and screen. In the experiment, the pictures taken by the camera is transmitted back to the camera lens again to create a simplest closed loop system. Therefore, the camera lens and the screen, built out of the Double Mirror Reflection model.

I did the experiments and saw some strange phenomena (Please see Appendix A).

\section{Results}

As Figures A1 and A2 shows, these mapped images simulate a double-mirror reflection model. It further instructs that if the brain has a similar structure, the singular phenomenon as camera-screen experiment shows will emerged in brain due to the continuous stimulation of neurons.

\section{Discussions}

According to the hypothesis, there are such mapping areas we called Brain Mirror Reflection Areas in the brain of the patients. In these areas the neurons would be repeatedly stimulated because of the information gotten by the sensory organs of patients which would lead to overgrowth of brain [5-7].

Recent study suggests the gene mutations which causes autism involves in the neuron development process [8]. It is also well-recognized that visual inputs or behavior activates specific areas of the patient's brain, and Jie Zhang and other researchers have experimentally found that autistic patients exhibit higher variability in specific brain areas [9]. The patients obtain some details of the information through some action, which will activate the brain mirror reflection area, thus the abnormal brain structures would lead abnormal behaviors, Then a positive feedback system is formed.

From the methodology of information ecology, we can use the idea of system to deal with the problem in details [10]. If this hypothesis model were proved, we can use the method of information ecology to try a way of treating autism by intervening information sources and information recipients, such as visual-brain intervention. By using the visual intervention we can control the visual, auditory, sensory inputs of autistic patients, and by using the brain intervention we can look for ROI (Region of interest) of patients, which we may call double mirror reflection areas, then the brain control equipment can be used on these areas to meddle their growth because of the neuron plasticity.

\section{Conclusions}

In this paper, the "double-mirror" hypothesis is proposed for autism, and it is thought that there are areas which have similar function like double mirror reflection. This is a non-linear iterative process, that is, a positive feedback process, which makes the information inputted into the 
brain repeatedly stimulate the relevant neurons and brain regions, resulting in the wrong connection and abnormal growth in the brain neurons of autistic patients, thus cause regrettable results.

In short, the study of autism spectrum disorders is still challenging, and need researchers from the basic and clinic to work together to continue to reveal the pathology of autism, to lay the foundation for further prevention and treatment of autistic.

Author Contributions: Jun Meng conceived the model and experiments; Xu Huang performed the experiments; Jun Meng and Xu Huang analyzed phenomena of the experiments; Xu Huang wrote the paper.

Conflicts of Interest: The authors declare no conflict of interest. The founding sponsors had no role in the design of the study; in the collection, analyses, or interpretation of data; in the writing of the manuscript, and in the decision to publish the results.

\section{Appendix A}

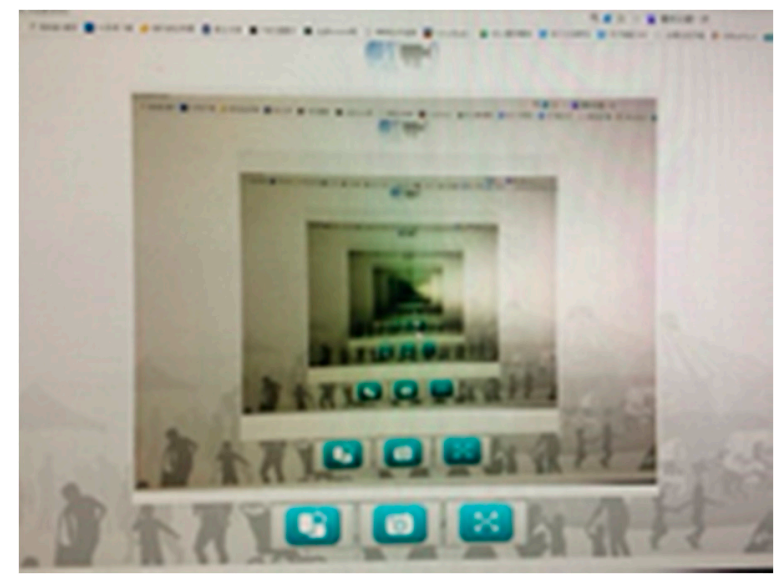

Figure A1. We actually use the computer monitor and mobile phone's web-camera to do the experiment. We shoot the computer screen through the lens, and the pictures the lens get will be transmitted to lens again. By repeating the iterative process, the mirror space in the "Double Mirror Reflection Model" is formed.

After the rotation of the camera, a lot of dazzling green, black, yellow and white phenomena were found in the screen.

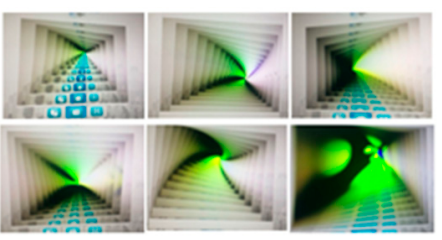

(a)

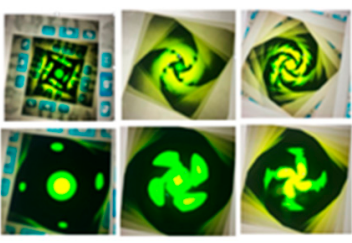

(b)

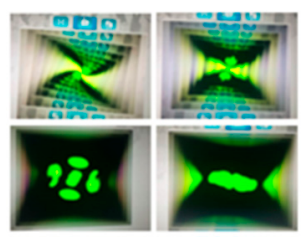

(c)

Figure A2. The image appears after the camera randomly rotation about (a) 0 degree; (b) 90 degrees; (c) 180 degrees. 


\section{References}

1. Association, A.P. Diagnostic and Statistical Manual of Mental Disorders (DSM-5 $\left.{ }^{\circledR}\right)$; America Psychiatric Pub: Washington, DC, USA, 2013.

2. Hebb, D.O. The Organization of Behavior: A Neuropsychological Theory; Psychology Press: East Sussex, UK, 2005.

3. Kolb, B.; Whishaw, I.Q. Brain plasticity and behavior. Ann. Rev. Psychol. 1998, 49, 43-64.

4. Curatolo, P.; Ben-Ari, Y.; Bozzi, Y.; Catania, M.V.; D’Angelo, E.; Mapelli, L.; Oberman, L.M.; Rosenmund, C.; Cherubini, E. Synapses as therapeutic targets for autism spectrum disorders: An international symposium held in Pavia on July 4th, 2014. Front. Cell. Neurosci. 2014, 8, 309.

5. Chawarska, K.; Campbell, D.; Chen, L.; Shic, F.; Klin, A.; Chang, J. Early generalized overgrowth in boys with autism. Arch. Gen. Psychiatry 2011, 68, 1021-1031.

6. Courchesne, E.; Karns, C.M.; Davis, H.R.; Ziccardi, R.; Carper, R.A.; Tigue, Z.D.; Chisum, H.J.; Moses, P.; Pierce, K.; Lord, C.; et al. Unusual brain growth patterns in early life in patients with autistic disorder an MRI study. Neurology 2001, 57, 245-254.

7. Hazlett, H.C.; Gu, H.;Munsell, B.C.; Kim, S.H.; Styner, M.; Wolff, J.J.; Elison, J.T.; Swanson, M.R.; Zhu, H.; Botteron, K.N.; et al. Early brain development in infants at high risk for autism spectrum disorder. Nature 2017, 542, 348-351.

8. Shao, S.; lin, C.; Quan, J.; Sen, L.; Feng, H. Advance in the Understanding of the Pathology of Autism on Synapse Dysfunction. Chin. J. Mod. Appl. Pharm. 2016, 33, 501-508.

9. Zhang, J.; Cheng, W.; Liu, Z.; Zhang, K.; Lei, X.; Yao, Y.; Becker, B.; Liu, Y.; Kendrick, K.M.; Lu, G.; et al. Neural, electrophysiological and anatomical basis of brain-network variability and its characteristic changes in mental disorders. Brain 2016, 139, 2307-2321.

10. ZiJing, H. Study on Information Ecology \& the Balance of Information Ecosystem. Master's Thesis, Zhejiang University, Hangzhou, China, 2008.

(C) 2017 by the authors. Licensee MDPI, Basel, Switzerland. This article is an open access article distributed under the terms and conditions of the Creative Commons Attribution (CC BY) license (http://creativecommons.org/licenses/by/4.0/) 